\title{
Production laitière de vaches Pie noire traites ou allaitant 3 veaux
}

\author{
O. PEREZ, N. JIMENEZ DE PEREZ, P. LE NEINDRE * \\ et J. COCHAUD
}

\author{
I.N.R.A., Station de Physiologie de la Reproduction, B.P. I, Nouzilly \\ F 37380 Monnaie \\ * C.R.Z.V. de Theix, Laboratoire de la Production de Viande \\ F 63122 Ceyrat
}

\begin{abstract}
Résumé
Trois lots de vaches multipares de race Frisonne Pie Noire sont étudiés. Celles des deux premiers lots allaitent chacune trois veaux, soit en liberté (lot AL : 16 vaches), soit sous surveillance deux fois par jour (lot AS : 19 vaches). Celles du troisième lot sont traites à la machine deux fois par jour (lot $T: 17$ vaches). Au cours de la lactation précédente, toutes ces vaches étaient traites et leurs productions laitières étaient voisines dans les trois lots. Durant la lactation expérimentale, les vaches allaitantes ont produit 40 p. 100 de lait de plus que les vaches traites, soit $25,9 \pm 3,4$ et $28,3 \pm 2,5$ contre $19,8 \pm 3,0 \mathrm{~kg}$ de lait par jour et par vache au cours des 4 premiers mois de la lactation pour les lots AL, AS et $\mathrm{T}$ respectivement. Lorsque le nombre de veaux est suffisant, l'allaitement permet une production laitière supérieure à celle obtenue à la traite, mais l'augmentation du nombre d'allaitements journaliers (de 2 pour le lot AS à 4 pour le lot AL) n'améliore pas la quantité de lait produite.
\end{abstract}

\section{Introduction}

La reconversion de vaches de races laitières ou mixtes de la traite à l'allaitement a été envisagée depuis longtemps, notamment parce que leur niveau de production permet d'allaiter simultanément plusieurs veaux en leur assurant des croissances élevées. Cette technique a fait l'objet de nombreux essais ou observations (Allemagne : WITT, 1960 ; Australie : Rowan \& Wall, 1970 ; Winks \& Edgley, 1970 ; Smith, Caliow \& MCSWeeney, 1973 ; Kaiser, 1975 ; Belgique : Boucque, Fiems \& Buysse, 1978 ; France : Petit \& Tartiere, 1971 ; Grenet, 1974 ; Irlande : Harte, 1970 ; Drennan, 1971 ; Nouvelle-Zélande : Everitt \& Philıpps, 1971).

Divers auteurs (Walsh, 1969 ; Le Neindre, Petit \& Muller, 1976) observent une augmentation de la production laitière de l'ordre de 15 à 26 p. 100 lorsque ces vaches passent de la traite à l'allaitement de plusieurs veaux. De même, des vaches laitières allaitant plusieurs veaux durant les 8 à 10 premières semaines de lactation et 
traites par la suite, produisent au-delà du sevrage de leurs veaux, plus de lait que leurs homologues traites depuis la mise bas (Everitt \& Phillips, 1971 ; Fulkerson, Hooley \& Findlay, 1978). Mais cette influence positive de l'allaitement sur la production n'est pas observée lorsque les vaches de race laitière ne nourrissent qu'un seul veau (Swanson, 1956).

Cependant, à partir de ces différents résultats bibliographiques, il n'est pas possible de préciser, d'une part les effets du mode d'extraction du lait (traite ou tétée) sur le niveau de production laitière et d'autre part ceux du nombre d'évacuations mammaires journalier, les vaches allaitant leurs veaux soit en liberté, soit deux fois par jour selon les cas. L'étude que nous présentons ici a permis de dissocier ces deux facteurs.

\section{Matériel et méthodes}

52 vaches multipares de race Frisonne Pie Noire du Domaine I.N.R.A. de Bressonvilliers ont été utilisées dans cet essai. Elles vêlent sur une courte période d'un mois, en majorité en septembre, et sont alors réparties en 3 lots : certaines sont traites à la machine (lot $T: 17$ vaches); les autres allaitent chacune 3 veaux, soit en liberté (lot $\mathrm{AL}$ : 16 vaches) soit sous surveillance deux fois par jour (lot AS : 19 vaches).

TABLEAU 1

Productions laitières journalières pendant les 110 premiers jours de lactation de vaches traites on allaitantes.

Daily milk production of milked or suckling cows during the first 110 days of lactation.

\begin{tabular}{|c|c|c|c|c|c|}
\hline \multirow[b]{2}{*}{$\begin{array}{l}\text { Mode de conduite } \\
\text { Management }\end{array}$} & \multirow{2}{*}{$\begin{array}{l}\text { Nombrc } \\
\text { de vaches } \\
\text { Number } \\
\text { of cows }\end{array}$} & \multicolumn{4}{|c|}{$\begin{array}{l}\text { Production journalière }(\mathrm{kg} / \text { jour }) \\
\text { Milk production }(\mathrm{kg} / \mathrm{d})\end{array}$} \\
\hline & & $\begin{array}{l}\text { A la traite } \\
\text { avant l'expérience } \\
\text { Before experiment } \\
\text { when milked }\end{array}$ & & $\begin{array}{r}\text { Pendant l'expé } \\
\text { During exI }\end{array}$ & $\begin{array}{l}\text { rimerstation } \\
\text { eriment }\end{array}$ \\
\hline $\begin{array}{l}\text { Allaitantes libres ..... } \\
\text { Suckling ad libitum } \\
\text { (lot AL) }\end{array}$ & 16 & $18,5 \pm 4,1$ & & $25,9 \pm 3,4$ & * \\
\hline $\begin{array}{l}\text { Allaitantes surveillées } \\
\text { Suckling twice daily } \\
\text { (lot AS) }\end{array}$ & 19 & $\left.\begin{array}{l|l}19,8 \pm 4,0 & \\
& \text { NS }\end{array}\right\}$ & NS & $28,3 \pm 2,5$ & ** \\
\hline $\begin{array}{l}\text { Traites } \ldots \ldots \\
\text { Milked } \\
(\operatorname{lot} \mathrm{T})\end{array}$ & 17 & $19,9 \pm 4,3$ & & $19,8=3,0$ & \\
\hline $\begin{aligned} \text { NS : } & \mathbf{P}>0,05 \\
(* *) & 0,05>\mathbf{P}>0,01 . \\
(* *) & \mathbf{P}<0,01 .\end{aligned}$ & & & & & \\
\hline
\end{tabular}


Au cours de la lactation précédente, où toutes les vaches étaient traites à la machine, les productions laitières moyennes des 3 lots étaient semblables (tabl. 1); il en était de même de leurs numéros de lactation (lot $\mathrm{AL}: 2,1 \pm 1,4$ de 1 à 5 ; lot $\mathrm{AS}$ : $2,4 \pm 1,2$ de 1 à 6 et lot $T: 2,5 \pm 1,6$ de 2 à 6 ).

\section{A. Lot $A L$ : vaches allaitantes «libres»}

Dès le vêlage, chaque vache est séparée de son veau en évitant tout contact entre les deux animaux. Le plus rapidement possible après le vêlage ( $66 \pm 59$ minutes), chaque. nourrice est enfermée dans un box de $16 \mathrm{~m}^{2}$ environ, avec 3 veaux étrangers de même âge pendant une période variant entre 10 et 20 jours selon le comportement des mères vis-à-vis des jeunes (en moyenne $15,5 \pm 2,7$ jours).

Après cette période, et jusqu'au sevrage des veaux (mois de mai-juin) à l'âge de 8 mois environ, les vaches sont rassemblées par groupe de 10 dans une stabulation libre, avec une aire commune aux vaches et aux veaux de $170 \mathrm{~m}^{2}$ et une zone de. $92 \mathrm{~m}^{2}$ réservée aux veaux.

\section{B. Lot $A S$ : vaches allaitantes «surveillées》}

Peu de temps après le vêlage (69 \pm 55 minutes), chaque vache passe une période de temps réduite ( $18 \pm 8$ heures) dans un box avec 3 veaux étrangers. Cette période n'a pas pour but d'assurer des adoptions, mais simplement de permettre à la vache d'établir un comportement maternel. Après cette période, les vaches et les veaux sont séparés, sauf lors des tétées journalières qui ont lieu sous surveillance vers 6 et 16 heures. Du fait de l'espace limité, ces tétées surveillées ont lieu ien deux temps; les 5 premières vaches et les 15 premiers veaux sortant de leurs parcs forment un premier groupe; les animaux restants en forment un second. Les vaches sont alors attachées au cornadis et les veaux peuvent choisir les vaches sous lesquelles ils vont téter. Une personne surveille les tétées matin et soir pour que chaque vache allaite effectivement 3 veaux et que ceux-ci ne changent pas de nourrice au cours de la tétée. Un égouttage manuel est dans quelques cas nécessaire au cours des premiers jours de la lactation.

\section{Lot $T$ : vaches traites}

Elles n'ont jamais de contact avec des veaux et sont traites à la machine 2 fois par jour vers 6 et 16 heures, dans une salle de traite en épi de marque Alfa-Laval de type Duovac. Les principales caractéristiques de la machine à traire sont les suivantes :

- niveau de vide : $42 \mathrm{kPa}$;

- Lactoduc ligne basse;

— vitesse de pulsation : $55 \mathrm{p} / \mathrm{min}$.

Il n'y a ni préparation de la mamelle à la traite ni égouttage, mais le système Duovac permet une dépression faible au début et à la fin de la traite.

\section{Alimentation des animaux}

Les mêmes quantités des différents aliments sont offertes aux vaches de chaque lot. Les quantités mentionnées sont exprimées en $\mathrm{kg}$ d'aliment par animal et par jour : 
- Alimentation pendant le mois précédant le vêlage : ensilage de graminée (4 à $5 \mathrm{~kg}$ de matière sèche), foin de graminée $(2 \mathrm{~kg})$, maïs et fétuque déshydratés $(8 \mathrm{~kg})$, aliment concentré $(1 \mathrm{~kg})$, tourteau de soja $(0,8 \mathrm{~kg})$.

- Alimentation après vêlage : maïs déshydraté $(10 \mathrm{~kg})$, luzerne déshydratée (3 kg), foin de graminée $(4 \mathrm{~kg})$, tourteau de soja $(0,65 \mathrm{~kg})$, aliment concentré $(2,5 \mathrm{~kg})$. Cette ration est calculée pour couvrir les besoins des vaches en considérant que la production laitière journalière est inférieure ou égale à $25 \mathrm{~kg}$.

Les quantités distribuées sont néanmoins approximatives car elles sont données à l'auge avec un distributeur mécanique (tracteur avec une remorque), et pour des groupes de 10 animaux. De plus, les veaux consomment une partie non connue des aliments distribués aux vaches du lot AL. Les veaux reçoivent en outre de l'aliment concentré ad libitum dans une case annexe.

\section{E. Mesure de la production laitière}

La production laitière des vaches traites est mesurée avant la fin du premier mois de lactation, puis à intervalle d'un mois environ jusqu'à la fin de la lactation.

L'estimation de la production laitière des allaitantes libres est faite par pesée des veaux avant et après la tétée matin et soir selon la technique proposée par LE NEINDRE (1973). En ce qui concerne les ailaitantes surveillées, les pesées sont effectuées lors des deux tétées journalières, les nourrices étant simplement tétées dans le même ordre le matin et le soir. Chez toutes les allaitantes, ces contrôles sont effectués mensuellement au cours des 4 premiers mois de lactation.

Les durées de lactation de certaines vaches allaitantes étant limitées à 110 jours, les productions laitières moyennes journalières sont calculées sur cette période.

Les comparaisons de la production laitière journalière entre lots sont réalisées à l'aide d'une analyse simple de la variance.

\section{Résultats}

Au cours de la lactation précédant l'expérience, où toutes les vaches étaient traites à la machine, les productions laitières n'étaient pas significativement différentes (tabl. 1), bien que les vaches du lot «allaitantes libres » aient produit à la traite un peu moins de lait que les vaches des deux autres lots.

Pendant l'expérience, les vaches allaitantes ont des productions laitières significativement supérieures, de 6 à $9 \mathrm{~kg}$, à celles des vaches traites (fig. 1 et tabl. 1). Enfin, la production laitière des vaches du lot AS est légèrement supérieure (de $2,4 \mathrm{~kg}$ en moyenne) à celle des animaux du lot $\mathrm{AL}(\mathrm{P}<0,05)$. Cela peut résulter partiellement du fait que leur production était légèrement plus élevée lors de la précédente lactation, mais l'introduction dans le modèle d'analyse d'un ajustement pour le niveau de production antérieure ne modifie pas le résultat. Aucune relation significative n'apparaît d'ailleurs entre les productions laitières obtenues au cours des deux lactations successives. 


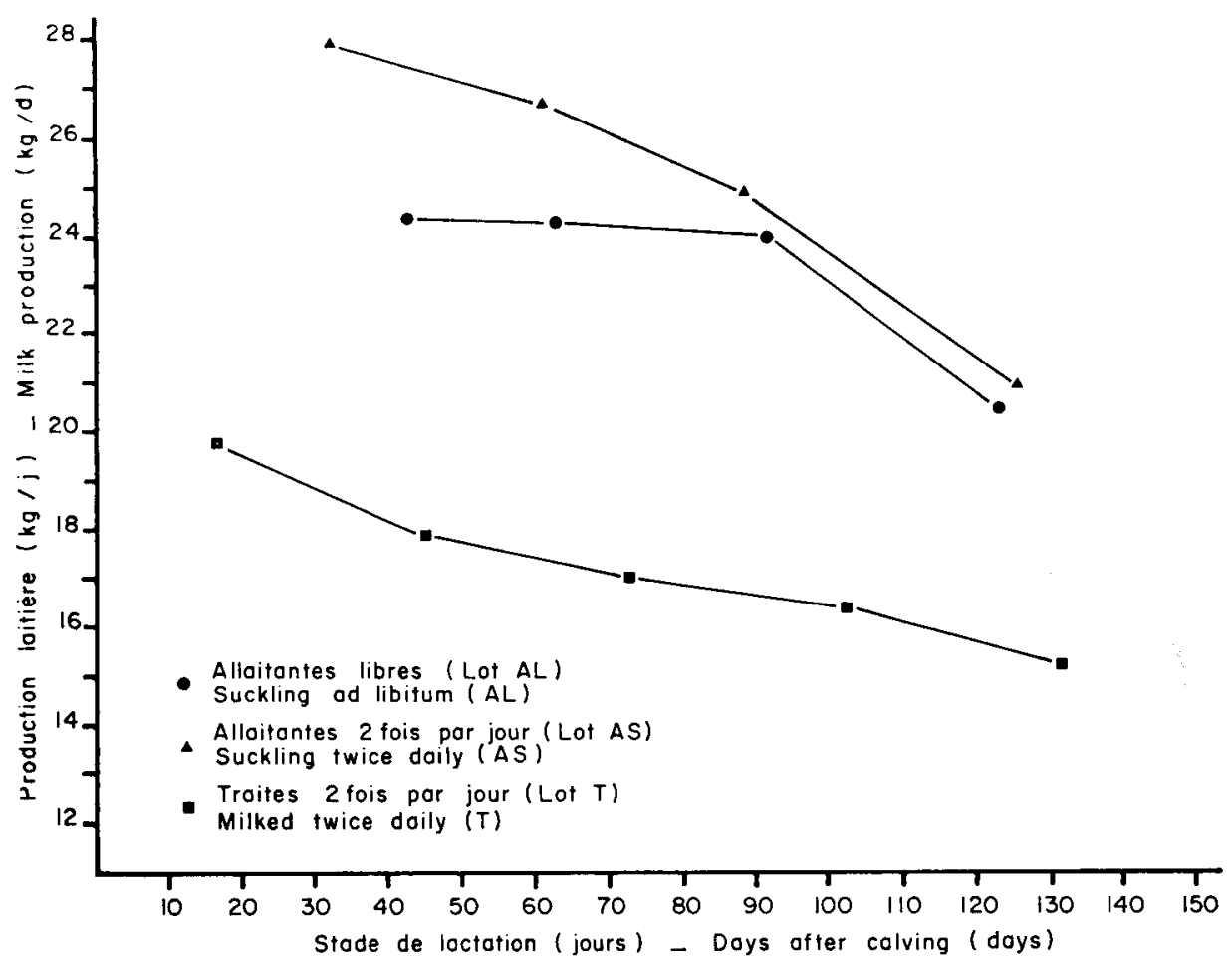

FIG. 1

Courbes de lactation des vaches des différents lots au cours de l'expérience.

Lactation curves during experiment.

\section{Discussion et conclusion}

Comme les divers auteurs (Everitt \& Phillips, 1971; Le Neindre, Petit \& Muller, 1976...) nous observons une augmentation très importante de la production laitière lorsque les vaches de race laitière passent de la traite à l'allaitement. Les vaches allaitantes ont eu une production laitière de 40 p. 100 supérieure à celles qui étaient traites, ce qui est plus important que l'écart habituellement observé notamment par Le Neindre, Petit \& Muller (1976) sur des vaches Normandes (+ 26 p. 100). Cela est peut-être dû à une meilleure réponse des vaches Frisonnes à l'allaitement mais plus probablement à une moindre qualité de la traite dans le présent essai, du fait de l'absence de préparation réelle de la mamelle à la traite et de l'absence d'égouttage.

Les travaux de Labussiere, Combaud \& Petrequin (1974) ont montré que chez des brebis Préalpes $d u S u d$, la tétée par un agneau étranger n'est pas plus efficace que la traite, et que seul le nombre journalier des évacuations mammaires modifie la 
production laitière. En revanche, les 3 veaux semblent plus efficaces que la machine à traire, soit par le fait d'une sécrétion plus importante des hormones galactopoiétiques, soit plutôt par une meilleure vidange de la mamelle résultant d'une meilleure efficacité de la succion des 3 veaux et/ou d'une sécrétion plus importante d'ocytocine (LE Neindre, Petit \& Muller, 1976).

L'éventuelle différence entre les deux espèces est peut-être liée à un comportement maternel plus sélctif des brebis par rapport aux vaches : les vaches de race laitière acceptent facilement de se laisser téter par des veaux étrangers (PoINDRon \& LE NEINDRE, 1979) alors que les brebis Préalpes du Sud refusent peut-être de donner leur lait à des agneaux étrangers comme à la machine à traire.

D'autre part, nous n'observons pas d'influence positive de l'augmentation du nombre journalier d'allaitements sur la production laitière des vaches quand on passe de 2 allaitements contrôlés à environ 4 allaitements par jour en liberté (Perez et al., non publié). En revanche, Labussiere, Combaud \& Petrequin (1974) obtiennent une augmentation de 20 p. 100 lorsque le nombre de tétées passe de 2 à 4 par jour chez les brebis. Cette différence entre espèces est peut-être due à une capacité de sécrétion de plus courte durée chez la brebis que chez la vache en l'absence de vidange, en liaison avec les fréquences naturelles de tétées beaucoup plus importantes chez la brebis. En effet, les agneaux tétent très fréquemment, de 15 tétées par période de 12 heures, à l'âge d'une semaine, à 7 tétées à l'âge de 8 semaines (EWBANK, 1967), alors que la fréquence des tétées des veaux diminue régulièrement de 6 à 3,5 tétées par jour entre 1 et 3 semaines, puis se maintient à ce niveau jusqu'à l'âge d'au moins 3 mois (Nicol \& Sharalfeldin, 1975).

Ces résultats non seulement confirment ceux obtenus antérieurement, mais montrent aussi que l'augmentation du nombre journalier d'allaitements chez la vache n'augmente pas (ou peu) la production laitière à l'inverse de ce qui a été observé par d'autres auteurs chez la brebis.

Accepté pour publication en juillet 1983.

\section{Summary \\ Milk production of Friesian cows milked or suckled by 3 calves}

Three groups of Friesian multiparous cows were studied. In the first two groups, each cow suckled three calves either ad libitum (group $\mathrm{AL}: 16$ cows) or twice daily under human control (group AS : 19 cows). Cows in the third group were milked twice daily (group $T: 17$ cows). Before the experiment when the cows were milked, no differences in milk production in the three groups were noted (tabl. 1). During experimental lactation, the milk production of the suckling cows (group AL : $25.9 \pm 3.4 \mathrm{~kg} / \mathrm{d}$, group AS : $28.3 \pm 2.5 \mathrm{~kg} / \mathrm{d}$ ) was significantly higher than that of milked cows (group $\mathrm{T}: 19.8 \pm 3.0 \mathrm{~kg} / \mathrm{d}$ ) (fig. 1). Suckling three calves was more efficient for milk production than milking. The increase of the daily number of suckling periods (group AL vs. group AS) was not followed by a new increase in milk production. 


\section{Références bibliographiques}

Boucque C.V., FIEMs L.O., Buysse F.X., 1978. Plusieurs veaux par vache allaitante de race pure à double fin. Rev. Agric., 31, 21-33.

Drennan M.J., 1971. Single-suckled beef production. II - Influence of stocking rate during the grazing season, creep grazing of the calf and double suckling on calf performance. Ir. J. agric. Res., 10, 297-305.

Everitt G.C., Phillips D.S.M., 1971. Calf rearing by multiple suckling and the effects on lactation performance on the cow. Proc. N.Z. Soc. Anim. Prod., 31, 22-40.

EWBank R., 1967. Nursing and suckling behaviour amongst Clun Forest ewes and lambs. Anim. Behav., 15, 25 I-258.

FUlKerson W.J., HoOleY R.D., Findlay J.K., 1978. Improvement of milk production of first calf heifers by multiple suckling. Aust. J. agric. Res., 29, 351-357.

Grenet N., 1974. Exemple d'utilisation des vaches laitières comme mères allaitantes. In : L'exploitation des troupeaux de vaches allaitantes. Bull. Tech. C.R.Z.V. Theix, I.N.R.A., $\mathrm{n}^{\circ}$ spécial, 307-321.

Harte F.J., 1970. Multiple suckling of calves. J. Ir. Grassl. and Anim. Prod. Assoc., 5, $65-78$.

KaISER A.G., 1975. Rearing dairy beef calves by multiple suckling. I - Elfects on liveweight change, onset of oestrus and post weaning milk production. Aust. J. Exp. Agric. Anim. Husb., 15, 17-24.

Labussiere J., Combaud J.F., Petrequin P., 1974. Influence de la fréquence des traites et des tétées sur la production laitière des brebis Préalpes du Sud. Ann. Zootech., 23, 445-457.

LE Neindre P., 1973. Observations sur l'estimation de la production laitièrc des vaches allaitantes par la pesće du veau avant et après la tétée. Ann. Zootech., 22, 413-422.

Le Neindre P., Petit M., Muller A., 1976. Production laitière de vaches Normandes à la traite ou à l'allaitement. Ann. Zootech., 25, 533-542.

Nicol A.M., Sharalfeldin M.A., 1975. Observations on the behaviour of single-suckled calves from birth to 120 days. Proc. N.Z. Soc. Anim. Prod., 35, 221-230.

Petit M., Tartiere H., 1971. Allaitement simultané de delix ou plusieurs veaux par vache. In : Production de viande par les jeunes bovins. Editions S.E.I., C.N.R.A. Versailles, étude $n^{\circ} 46,65-72$.

Poindron P., Le Neindre: P., 1979. Les relations mère-jeune chez les ruminants domestiques et leurs conséquences en production animale. Bull. Tech. Dep. Génét. anim., 29-30, pp. 33-57.

Rowan K.J., Wal.L P.F., 1970. A study of suckling of calves in pairs for vealer production. Proc. Aust. Soc. Anim. Prod., 1970, 243-246.

Smith M.E., Callow C., McSweeney B.J., 1973. Ten and eighteen-week suckling of friesian steers. Proc. N.Z. Soc. Anim. Prod., 33, 161-175.

Swanson E.W., 1956. The effect of nursing calves on milk production of identical twin heifers. J. Dairy Sci., 39, 73-80.

WALSH J.P., 1969. Effect of suckling in early and late lactation on the milk yield of cow. An Foras Taluntais, Animal Production Research Report, p. 24 (Abstr.).

WiNKs L., Edgley W., 1970. Multiple suckling of calves on culled dairy cows grazing Green Panic-Glycine Wightii pasture. Proc. 18th. Int. Dairy Congr. Sydney, 1, 688.

Witт M., 1960. Ammenkühe für Aufzucht und Mastzwecke. Landwirtschaftsblatt WeserEms., Nr. 45 und 46. 